\title{
Recovery of muscle function after deep neuromuscular block by means of diaphragm ultrasonography and adductor of pollicis acceleromyography with comparison of neostigmine vs. sugammadex as reversal drugs: study protocol for a randomized controlled trial
}

lacopo Cappellini ${ }^{*} \mathbb{D}$, Fabio Picciafuochi, Daniele Ostento, Ginevra Danti, Angelo Raffaele De Gaudio and Chiara Adembri

\begin{abstract}
Background: The extensive use of neuromuscular blocking agents (NMBAs) during surgical procedures still leads to potential residual paralyzing effects in the postoperative period. Indeed, neuromuscular monitoring in an intra-operative setting is strongly advocated. Acetylcholinesterase inhibitors can reverse muscle block, but their short half-life may lead to residual curarization in the ward, especially when intermediate or long-acting NMBAs have been administered. Sugammadex is the first selective reversal drug for steroidal NMBAs; it has been shown to give full and rapid recovery of muscle strength, thus minimizing the occurrence of residual curarization. Acceleromyography of the adductor pollicis is the gold standard for detecting residual curarization, but it cannot be carried out on conscious patients. Ultrasonography of diaphragm thickness may reveal residual effects of NMBAs in conscious patients.

Methods/design: This prospective, double-blind, single-center randomized controlled study will enroll patients (of American Society of Anesthesiologists physical status I-II, aged 18-80 years) who will be scheduled to undergo deep neuromuscular block with rocuronium for ear, nose, or throat surgery. The study's primary objective will be to compare the effects of neostigmine and sugammadex on postoperative residual curarization using two different tools: diaphragm ultrasonography and acceleromyography of the adductor pollicis. Patients will be extubated when the train-of-four ratio is $>0.9$. Diaphragm ultrasonography will be used to evaluate the thickening fraction, which is the difference between the end expiratory thickness and the end inspiratory thickness, normalized to the end expiratory thickness. Ultrasonography will be performed before the initiation of general anesthesia, before extubation, and 10 and 30 min after discharging patients from the operating room. The secondary objective will be to compare the incidence of postoperative complications due to residual neuromuscular block between patients who receive neostigmine and those who receive sugammadex.

(Continued on next page)
\end{abstract}

\footnotetext{
* Correspondence: jacopocappellini@gmail.com; iacopo.cappellini@unifi.it Department of Health Sciences, Section of Anesthesiology and Critical Care, University of Florence, Largo Brambilla 3, 50134 Florence, Italy
} 
(Continued from previous page)

Discussion: Postoperative residual curarization is a topic of paramount importance, because its occurrence can cause complications and increase the length of stay in hospital and the related costs. Diaphragm ultrasound assessment may become a bedside integrative tool in the neuromuscular monitoring field to detect concealed residual curarization in surgical patients who have received paralyzing agents.

Trial registration: EudraCT, 2013-004787-62. Registered on 18 June 2014, as "Evaluation of muscle function recovery after deep neuromuscular blockade by acceleromyography of the adductor pollicis or diaphragmatic echography: comparison between sugammadex and neostigmine."

ClinicalTrials.gov, NCT02698969. Registered on 15 February 2016, as "Recovery of Muscle Function After Deep Neuromuscular Block by Means of Diaphragm Ultrasonography and Adductor Pollicis Acceleromyography: Comparison of Neostigmine vs. Sugammadex as Reversal Drugs."

Keywords: Diaphragm ultrasonography, Sugammadex, Postoperative residual, Curarization, Neuromuscular monitoring,

\section{Background}

Non-depolarizing neuromuscular blocking agents (NMBAs) are extensively used by anesthesiologists to maintain deep neuromuscular block (dNMB) during surgical operations. To avoid the postoperative residual effects of NMBAs, muscle relaxants should be fully catabolized to inactive metabolites prior to extubation. Nevertheless, when a patient is waking from general anesthesia, it is possible that some of the administered paralyzing agent is not completely transformed to its inactive form at the level of neuromuscular junctions, causing residual effects that can be difficult to clinically diagnose without adequate neuromuscular monitoring [1].

The use of intra-operative neuromuscular monitoring when NMBAs are administered has been encouraged in order to decrease postoperative residual curarization (PORC) [2]. Acceleromyography, the most commonly used method of quantitative monitoring, appraises muscle acceleration responding to nerve stimulation by train-of-four (TOF) and post-tetanic count (PTC) methods [3]. For many years, a TOF ratio not greater than 0.9 between the amplitude of the last stimulation and that of the first one was used to define PORC. Despite the fact that these types of monitoring are strongly recommended, they are not regularly performed in the operating room scenario $[4,5]$. Furthermore, the TOF test is unpleasant for patients when they are conscious.

The incidence of PORC ranges from 9 to $56.5 \%$ when no reversal drug is administered [6]. For many years, reversing the NMBA effect has been carried out using an acetylcholinesterase inhibitor (AChEI) such as neostigmine. These inhibitors, which increase acetylcholine levels in the neuromuscular junction, antagonize the paralyzing agent but do not hasten its metabolism. Therefore, as a result of the unpredictable metabolism of blocking drugs, a residual curarization may occur when the AChEI effect has elapsed [7]. Indeed, an observational study showed that, 20 min after administration of neostigmine, $18 \%$ of patients had a TOF ratio $<0.9$ [8]. Moreover, as shown in an animal study, when neostigmine is administered in the setting of full recovery from a muscle relaxant, the drug can cause weakness of the diaphragm and genioglossus muscle even if this effect is not seen when residual curarization is still present [9].

Sugammadex is the first selective reversal agent for steroidal NMBAs. It has been shown to give full and rapid recovery of muscle strength, thus minimizing the occurrence of PORC $[8,10]$.

The diaphragm, the major respiratory muscle in humans, is a great septum between the thoracic and abdominal cavities. The movement of the diaphragm accounts for $60-70 \%$ of the total tidal volume of respiration. Failure of diaphragmatic function is believed to play a central role in the pathophysiology of the clinical syndrome known as "pump respiratory failure" [11-13]. Although a TOF ratio $>0.9$ in the adductor pollicis rules out residual curarization, the diaphragm is often not evaluated in the operating room. The diaphragm is the most highly resistant muscle to NMBAs, as well as the first to recover [14], but the occurrence of its dysfunction has been implicated in postoperative respiratory failure, especially when mechanical ventilation is prolonged $[15,16]$. Therefore, studying diaphragmatic function in a perioperative context is extremely important.

Since 1985, ultrasonography has been used to evaluate diaphragm function by measuring thickness variations in the apposition zone, which reflect the extent of contraction of the muscle [17]. Vivier et al. recently demonstrated that the thickening fraction (TF), namely the difference between the thickness at the end of inspiration (TEI) and that at the end of expiration (TEE), normalized for TEE (TEI - TEE/TEE), is directly related to respiratory workload, and they suggested that TF could be used as an index to select those patients ready to be weaned from noninvasive ventilation [18]. These data suggest that ultrasound TF could also be used in different scenarios, and 
our purpose is to use ultrasound TF to assess diaphragm recovery after $\mathrm{dNMB}$ since it is more comfortable for conscious patients than acceleromyography.

We therefore hypothesize that the incidence of postoperative diaphragmatic dysfunction, assessed using ultrasonography and, from our unpublished observations, defined by a fractional shortening of the diaphragm $<40 \%$, is lower in patients who receive sugammadex than in those who receive neostigmine.

\section{Methods/design}

\section{Study design and eligibility}

This study is a prospective, double-blind, randomized controlled trial involving 60 patients with American Society of Anesthesiologists (ASA) physical status I-II and aged between 18 and 80 years, who will undergo dNMB (standard care in our clinic) with rocuronium during ear, nose, or throat surgery in a university hospital. The Institutional Review Board of the Tuscany Region has approved the protocol with registration number CE SPE 13.068. Exclusion criteria are a history of hepatic or renal disease, chronic or acute alcoholism, allergy or hypersensitivity to sugammadex or neostigmine, current medication with effects on the central nervous system, a history of neurologic disease, diaphragmatic palsy, women who are pregnant or nursing, and arrhythmias.

\section{Randomization}

Written informed consent will be obtained during the preoperative evaluation by an anesthesiologist working in the anesthesia unit of the hospital. Afterwards, each patient will be randomly allocated to either the sugammadex (SUG) group or the neostigmine (NEO) group. Randomization will be performed using a table created on www.randomization.com. The allocation plan will be carried out using a variable block randomization method 1:1 to distribute the patients equally to each group. For allocation concealment, table assignment to one group or the other will be managed by a pharmacist with limited involvement in the study; this person will also perform the allocation and prepare the drugs.

\section{Intervention plan}

In order to standardize the anesthetic technique, no premedication will be administered. All patients will undergo neuromuscular monitoring with ulnar nerve stimulation using the TOF-Watch (Organon, Oss, Netherlands). The device will be calibrated preoperatively, and the parameters will be set using standard TOF methodology after administration of a hypnotic drug, prior to muscle relaxation. General anesthesia will be induced by intravenous injection of fentanyl ( $2 \mu \mathrm{g} / \mathrm{kg}$ body weight), propofol $(2 \mathrm{mg} / \mathrm{kg})$, and rocuronium $(0.6 \mathrm{mg} / \mathrm{kg})$. Tracheal intubation will be performed after the patient fails to register signals using TOF. To maintain $\mathrm{dNMB}$, rocuronium $(0.15 \mathrm{mg} / \mathrm{kg})$ will be re-administered when the PTC elicits more than five twitches. Sevoflurane will be supplied at an age-adjusted end-tidal concentration of 1.0 minimum alveolar concentration (MAC) in an air/oxygen mixture. Fentanyl will be titrated with a bolus of $0.5 \mu \mathrm{g} / \mathrm{kg}$ every $30 \mathrm{~min}$, to keep an adequate level of analgesia.

Prior to induction of anesthesia, the baseline TF will be evaluated by one operator skilled in ultrasonography using an Esaote MyLab ultrasound instrument (Esaote, Genoa, Italy). Patients will be placed on the bed in a semi-recumbent $\left(45^{\circ}\right)$ position, assessed by a goniometer. The operator will use a $10-12 \mathrm{MHz}$ highfrequency linear probe to identify the diaphragm in the midaxillary line in the apposition zone between the lung and liver on the right and between the lung and spleen on the left, in the intercostal spaces between the ninth, tenth, and eleventh ribs, $0.5-2 \mathrm{~cm}$ above the costophrenic sinus. The TF will be calculated at tidal breathing, recorded in time-motion mode. The muscle will be located using the hyperechoic pleural and peritoneal layers. Three assessments will be performed in consecutive breaths and averaged [18].

At the end of the operation and when TOF neuromuscular monitoring shows a minimum of two twitches, patients will receive the reversal drug according to the group to which they have been randomized. Patients in the NEO group will receive $50 \mu \mathrm{g} / \mathrm{kg}$ neostigmine and $15 \mu \mathrm{g} / \mathrm{kg}$ atropine, while those in the SUG group will receive $2 \mathrm{mg} / \mathrm{kg}$ sugammadex [19]. The drugs will be prepared for intravenous injection in identical volumes and in indistinguishable syringes so that the anesthesiologist will be blinded to the treatments the patients receive. Extubation will be performed when all the following criteria are met: (1) the patient is awake and can execute simple commands; (2) the patient's respiratory pattern is regular with a tidal volume of 6-7 mL/kg referred to ideal body weight; (3) the TOF ratio is $>0.9$. Immediately prior to extubation, bilateral diaphragm ultrasonography will be performed to assess muscle recovery in spontaneously breathing patients; these measurements will be compared to the baseline muscle assessment. Two additional diaphragm ultrasound scans will be performed 10 and $30 \mathrm{~min}$ after discharge from the operating theater under the same conditions as described above, but no further TOF monitoring will be carried out. Follow-up will be performed to document adverse events and complications until discharge from the hospital. The physician who performs the ultrasound scan will be different from the one who administers the reversal drug and will be blinded to the treatment that patients receive (Figs. 1 and 2). The Standard Protocol Items: Recommendations for Interventional Trials (SPIRIT) checklist is provided as Additional file 1 . 


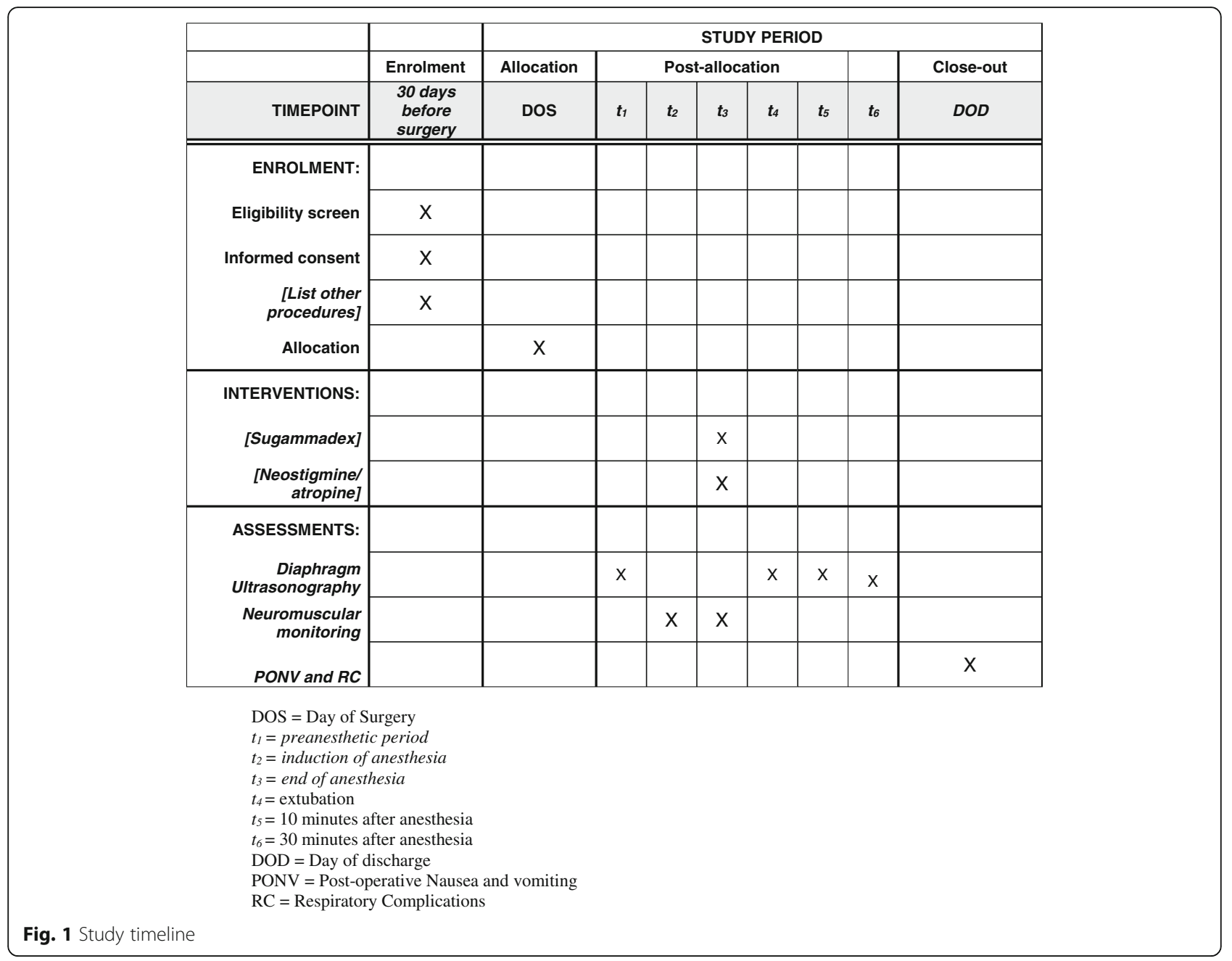

In the case of unexpected events, such as a change in drug dose or a participant's request to withdraw from the study, the protocol will be stopped and this will be recorded on the case report form (CRF). No further tests will be performed except those necessary to finalize the protocol. Should patients need medical care that interferes with the correct conduct of the study, they will be excluded from the study.

The primary endpoint of the study will be a $30 \%$ relative reduction in the incidence of residual curarization in patients who receive sugammadex compared with neostigmine $30 \mathrm{~min}$ after drug administration. The primary endpoint will be assessed at $30 \mathrm{~min}$ because the effects of neostigmine start to fade after this time has elapsed [20]. Residual curarization will be determined by the percentage of $30 \mathrm{~min}$ TF compared to baseline TF. It is not known exactly what percentage TF indicates residual curarization, but from our unpublished observations, values of $40 \%$ or less could indicate diaphragmatic dysfunction. However, we do not know what percentage of patients have a $\mathrm{TF}<40 \%$ when neostigmine is administered.

Two secondary endpoints will be assessed. One secondary endpoint will be a $10 \%$ relative reduction in respiratory complications related to residual curarization obtained with sugammadex compared with neostigmine. Respiratory complications taken into consideration will be new cough and sputum production, abnormal breath sounds not present at baseline, temperature higher than $38{ }^{\circ} \mathrm{C}$, chest radiography documentation of atelectasis or new infiltrates, and physician documentation of atelectasis or pneumonia [21]. The other secondary endpoint will be a $30 \%$ relative decrease in postoperative nausea or vomiting in patients who receive sugammadex compared with those who receive neostigmine.

Data monitoring will be performed by an anesthetist not involved in the study. Data will be collected on paper CRFs. All personal information will be registered in an environment limited to medical personnel to maintain absolute confidentiality. Data entry will be performed at one 


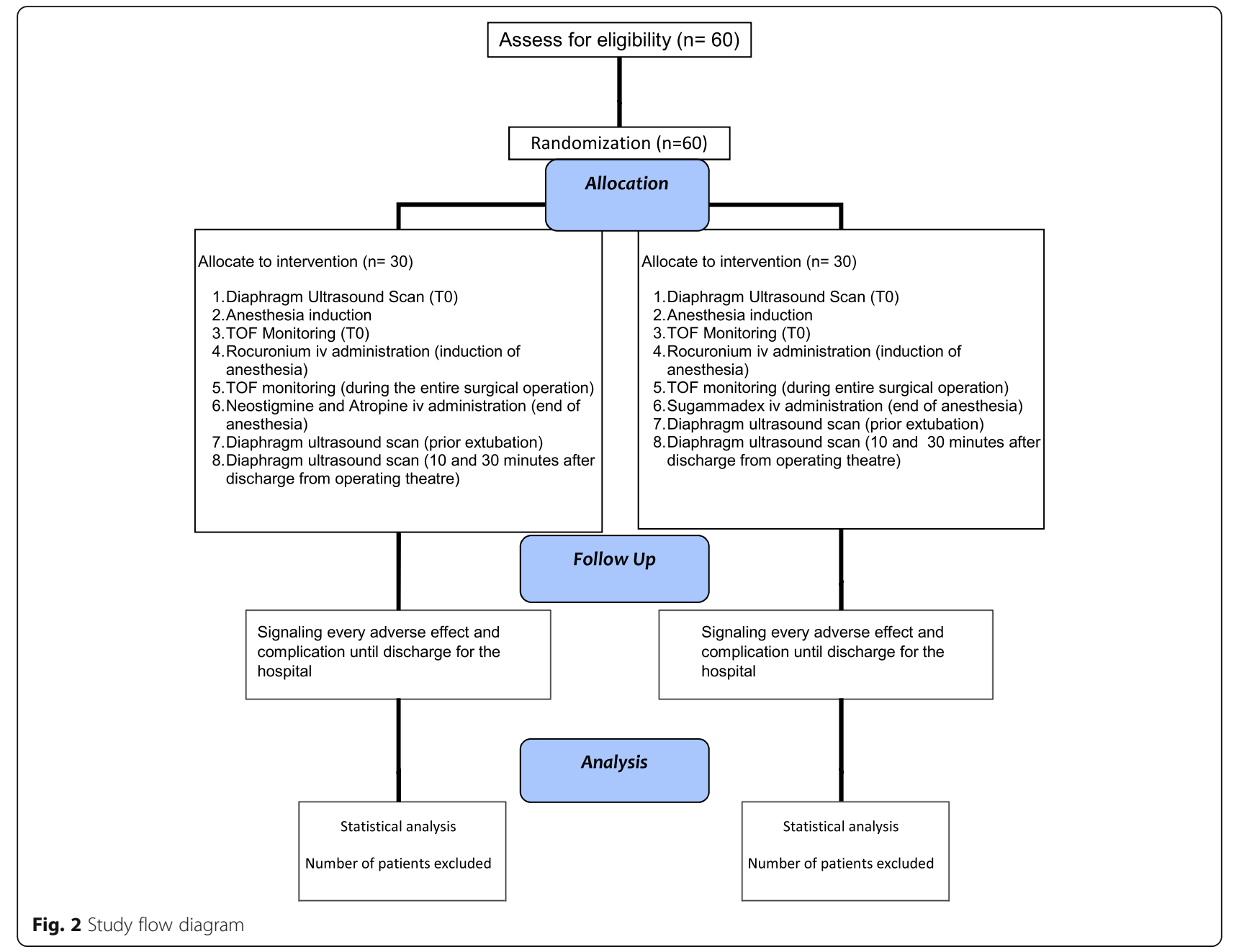

central site that maintains the overall database and will carry out the data analysis. All the compiled CRFs will be archived in a locker to which only clinicians involved in the study have access. In order to eliminate possible data entry errors, individual data will be compared to a range of plausible values. After data entry, automated checks, which have been defined a priori, will be performed to search for internal inconsistencies, range errors, or missing data. For each atypical, out-of-range, or missing datum, a query will be automatically sent to the investigator. Once all the queries are solved, the database will be locked and used for statistical analysis. All the individual participant data collected during the trial, after deidentification, will be available. The study protocol, statistical analysis plan, and analytic code will be accessed beginning 3 months and ending 5 years following article publication by researchers who will provide a methodologically sound proposal to achieve aims in the approved proposal. Each request must be sent to the corresponding author.

\section{Statistical analyses and sample size calculation}

The statistical analysis will be performed by an independent statistician using SAS 9.3 (SAS Institute, Inc., Cary, NC, USA). For the primary endpoint, the effect of a drug on $\triangle T F$ will be estimated using generalized estimating equations (GEEs) and a multiple linear regression model adjusting for time of the measurement and baseline TF of the right side. This hemidiaphragm has been chosen because it is more easily identified for the presence of the liver. For the secondary endpoint, the association between a drug and its collateral effect will be evaluated using a logistic regression model. $P$ values lower than 0.05 have been considered statistically significant.

Finally, descriptive statistics of all variables describing the characteristics of the patients enrolled in the study and those excluded from the study will be analyzed. Continuous variables will be expressed as mean ( \pm standard deviation, (SD)) and median (ranging from 25th to 75th percentiles). Percentages will be 
calculated for dichotomous data. For categorical variables, frequency counts and percentages will be calculated.

Since this is the first clinical trial that proposes, as its primary endpoint, a relative reduction of $30 \%$ in the incidence of residual curarization, the necessary sample size has been calculated using the statistical software Epi Info (version 7). This analysis shows that at least 30 patients per group will be necessary, considering that in $5 \%$ of subjects TF is not valuable $[22,23]$, and expecting $23-25 \%$ of residual curarization with neostigmine $30 \mathrm{~min}$ after the extubation vs. $2-4 \%$ after the administration of sugammadex (with a $95 \%$ confidence interval $(\mathrm{CI})$ and a power of $80 \%$, and assuming equal variance between the two groups). For the secondary outcome, the number needed to treat has been calculated with a CI of $95 \%$.

\section{Discussion}

Diaphragm ultrasonography has been used for 25 years to evaluate diaphragmatic dysfunction in many clinical scenarios [18, 24-26]. Ultrasound assessment of the diaphragm is not feasible if the operator is not adequately trained, but when skilled operators are available, this tool enables bedside evaluation of the major respiratory muscle. We have observed (unpublished observations) that the reproducibility and repeatability of diaphragm ultrasonography are moderate when the test is performed by three different operators with different levels of experience with sonography. However, one potential limitation of the present protocol could be that previous studies have demonstrated that repeatability ranges from 13 to $19 \%$ [22].

This study will be the first to assess if TF measurements in the operating room enable physicians to diagnose and eventually treat residual curarization after dNMB. The rationale for comparing sugammadex to neostigmine is that the latter has a pharmacokinetic profile that cannot avoid residual curarization, especially when an intermediate or long-lasting muscle relaxant is administered during general anesthesia, while the former avoids residual curarization because it binds stably with steroid NMBA molecules by means of van der Waals and hydrophobic interactions [27]. Therefore, the present study will be the first that aims to detect diaphragmatic dysfunction using ultrasonography for the purpose of assessing PORC after deep neuromuscular blockade with an aminosteroid muscle relaxant drug.

\section{Trial status}

Currently, patient recruitment is completed and all data have been collected. Data analysis will begin shortly.

\section{Additional file}

Additional file 1: SPIRIT 2013 checklist: recommended items to address in a clinical trial protocol and related documents. (DOC $122 \mathrm{~kb}$ )

\section{Abbreviations \\ AChEl: Acetylcholinesterase inhibitor; ASA: American Society of Anesthesiologists; Cl: Confidence interval; CRF: Case report form; dNMB: Deep neuromuscular block; MAC: Minimum alveolar concentration; NEO: Neostigmine; NMBA: Neuromuscular blocking agent; \\ PORC: Postoperative residual curarization; PTC: post-tetanic count; SUG: Sugammadex; TEE: Thickness at the end of expiration; TEl: Thickness at the end of inspiration; TF: Thickening fraction; TOF: Train of four}

\section{Acknowledgements}

Not applicable.

\section{Funding}

This study has been supported only in part by a research grant from the Investigator-Initiated Studies Program of Merck Sharp \& Dohme Corp. (MSD) with the reference number MISP 53534. The opinions expressed in this paper are those of the authors and do not necessarily represent those of MSD. MSD has not been involved in any of the following parts of the study: study design, data collection, data management, analysis, and interpretation of data. A final report will be furnished once the enrollment is completed. Also, MSD has no ultimate authority over the above-mentioned activities. The authors will independently decide whether and where the report will be suitable for possible publication. The rest of the research funds have been provided by the University of Florence, Department of Health Sciences, Section of Anesthesiology and Critical Care.

\section{Availability of data and materials}

Data will be collected using paper CRFs. Data entry will be performed at one central site that maintains the overall database and will carry out the data analysis. The database will be available for editors and peer reviewers, if required. All the compiled CRFs will be archived. In order to eliminate possible data entry errors, individual data will be compared to a range of plausible values. After data entry, automated checks, which will be defined a priori, will be performed to search for internal inconsistencies, range errors, or missing data. For each atypical, out-of-range, or missing datum, a query will be automatically sent to the investigators. Once all the queries are solved, the database will be locked and used for statistical analysis.

\section{Authors' contributions}

IC, FP, DO, GD, ARDG, and CA contributed substantially to the study design. IC drafted the manuscript. IC, FP, DO, GD, ARDG, and CA revised and approved the final version before submission. All authors read and approved the final manuscript.

\section{Ethics approval and consent to participate}

Written informed consent will be obtained from all participants. The Comitato Etico Regione Toscana - Area Vasta Centro, the local institutional review board, approved the present protocol of study with registration number CE SPE 13.068.

\section{Consent for publication}

Written informed consent will be obtained from each participant for publication of their individual details and accompanying images in this manuscript. The consent form is held by the authors.

Competing interests

The authors declare that they have no competing interests.

\section{Publisher's Note}

Springer Nature remains neutral with regard to jurisdictional claims in published maps and institutional affiliations. 
Received: 27 March 2017 Accepted: 5 February 2018

\section{Published online: 21 February 2018}

\section{References}

1. Viby-Mogensen J. Postoperative residual curarization and evidence-based anesthesia. Br J Anaesth. 2000;84(3):301-3.

2. Naguib M, Kopman AF, Ensor JE. Neuromuscular monitoring and postoperative residual curarisation: a meta-analysis. Br J Anaesth. 2007;98(3): 302-16.

3. Samet A, Capron F, Alla F, Meistelman C, Fuchs-Buder T. Single acceleromyographic train-of-four, 100-Hertz tetanus or double-burst stimulation: which test performs better to detect residual paralysis? Anesthesiology. 2005;102(1):51-6.

4. Teoh WH, Ledowski T, Current TPS. trends in neuromuscular blockade, management, and monitoring amongst Singaporean anaesthetists. Anesthesiol Res Pract. 2016;2016:7284146.

5. Phillips $S$, Stewart $P$, Bilgin A. A survey of the management of neuromuscular blockade monitoring in Australia and New Zealand. Anaesthesia Intens Care. 2013;41:374-9.

6. Aytac I, Postaci A, Aytac B, Sacan O, Alay GH, Celik B, et al. Survey of postoperative residual curarization, acute respiratory events and approach of anesthesiologists. Rev Bras Anestesiol. 2016;66(1):55-62.

7. Magorian TT, Lynam DP, Caldwell JE, Miller RD. Can early administration of neostigmine, in single or repeated doses, alter the course of neuromuscular recovery from a vecuronium-induced neuromuscular blockade? Anesthesiology. 1990;73:410-4

8. Della Rocca G, Pompei L, de Paganis CP, Tesoro S, Mendola C, Boninsegni P, et al. Reversal of rocuronium induced neuromuscular block with sugammadex or neostigmine: a large observational study. Acta Anaesthesio Scand. 2013;57(9):1138-45.

9. Eikermann M, Fassbender P, Malhotra A, Takahashi M, Kubo S, Jordan AS, et al. Unwarranted administration of acetylcholinesterase inhibitors can impair genioglossus and diaphragm muscle function. Anesthesiology. 2007;107(4): 621-9.

10. Flockton EA, Mastronardi P, Hunter JM, Gomar C, Mirakhur RK, Aguilera L, et al. Reversal of rocuronium-induced neuromuscular block with sugammadex is faster than reversal of cisatracurium-induced block with neostigmine. $\mathrm{Br}$ J Anaesth. 2008;100(5):622-30,

11. Berdah SV, Picaud R, Jammes Y. Surface diaphragmatic electromyogram changes after laparotomy. Clin Physiol Funct Imaging. 2002;22(2):157-60.

12. Mead J, Loring SH. Analysis of volume displacement and length changes of the diaphragm during breathing. J Appl Physiol. 1982;53(3):750-5.

13. Ford GT, Whitelaw WA, Rosenal TW, Cruse PJ, Diaphragm GCA. function after upper abdominal surgery in humans. Am Rev Respir Dis. 1983;127(4):431-6.

14. Nguyen-Huu T, Molgó J, Servent D, Duvaldestin P. Resistance to Dtubocurarine of the rat diaphragm as compared to a limb muscle: influence of quantal transmitter release and nicotinic acetylcholine receptors. Anesthesiology. 2009;110(5):1011-5.

15. Levine S, Nguyen T, Taylor N, Friscia ME, Budak MT, Rothenberg P, et al. Rapid disuse atrophy of diaphragm fibers in mechanically ventilated humans. N Engl J Med. 2008;358(13):1327-35.

16. Cho H, Kim S, Jung S, Kim S, Ma J, Yu D, et al. Effects of lower tidal volume on ventilator-induced diaphragmatic dysfunction. J Lung Pulm Respir Res. 2017:4:1-7.

17. Fried AM, Cosgrove DO, Nassiri DK, McCready VR. The diaphragmatic echo complex: an invitro study. Investig Radiol. 1985;20(1):62-7.

18. Vivier E, Mekontso Dessap A, Dimassi S, Vargas F, Lyazidi A, Thille AW, et al. Diaphragm ultrasonography to estimate the work of breathing during noninvasive ventilation. Intensive Care Med. 2012;38(5):796-803.

19. Kirkegaard-Nielsen H, Helbo-Hansen HS, Lindholm P, Severinsen IK, Time BK. to peak effect of neostigmine at antagonism of atracurium- or vecuroniuminduced neuromuscular block. J Clin Anesth. 1995;7(8):635-9.

20. Zhang B, Hepner D, Tran MH, Friedman M, Korn JR, Neuromuscular MJ. blockade, reversal agent use, and operating room time: retrospective analysis of US inpatient surgeries. Curr Med Res Opin. 2009;25(4):943-50.

21. JA BB. Predictors of postoperative pulmonary complications following abdominal surgery. Chest. 1997;111(3):564-71.

22. Goligher EC, Laghi F, Detsky ME, Farias P, Murray A, Brace D, et al. Measuring diaphragm thickness with ultrasound in mechanically ventilated patients: feasibility, reproducibility and validity. Intensive Care Med. 2015; 41(4):642-9.
23. Green MS, Venkatesh AG, Venkataramani R. Management of residual neuromuscular blockade recovery: age-old problem with a new solution. Case Rep Anesthesiol. 2017;2017:8197035.

24. Kim WY, Suh HJ, Hong S-B, Koh Y, Lim C-M. Diaphragm dysfunction assessed by ultrasonography: influence on weaning from mechanical ventilation. Crit Care Med. 2011;39(12):2627-30.

25. Grosu HB, Lee Yl, Lee J, Eden E, Eikermann M, Rose KM. Diaphragm muscle thinning in patients who are mechanically ventilated. Chest. 2012;142(6):1455-60.

26. DiNino E, Gartman EJ, Sethi JM, McCool FD. Diaphragm ultrasound as a predictor of successful extubation from mechanical ventilation. Thorax. 2013;69:1-5.

27. Adam JM, Bennett DJ, Bom A, Clark JK, Feilden H, Hutchinson EJ, et al. Cyclodextrin-derived host molecules as reversal agents for the neuromuscular blocker rocuronium bromide: synthesis and structure-activity relationships. J Med Chem. 2002;45(9):1806-16.

\section{Submit your next manuscript to BioMed Central and we will help you at every step:}

- We accept pre-submission inquiries

- Our selector tool helps you to find the most relevant journal

- We provide round the clock customer support

- Convenient online submission

- Thorough peer review

- Inclusion in PubMed and all major indexing services

- Maximum visibility for your research

Submit your manuscript at www.biomedcentral.com/submit
C Biomed Central 